\title{
Complexity and wide range of neuromyelitis optica spectrum disorders: more than typical manifestations
}

This article was published in the following Dove Press journal:

Neuropsychiatric Disease and Treatment

20 October 2017

Number of times this article has been viewed

\author{
Jinming Han' \\ Meng-ge Yang' \\ Jie Zhu',2 \\ Tao Jin'
}

'Department of Neurology and Neuroscience Center, The First Hospital of Jilin University, Changchun, China; ${ }^{2}$ Department of Neurobiology, Care Sciences and Society, Karolinska Institute, Stockholm, Sweden
Correspondence: Tao Jin Department of Neurology and Neuroscience Center, The First Hospital of Jilin University, 7I Xinmin Street, Changchun I3002I, China

Tel +8688782378

Emaildrtao.jin@hotmail.com

\begin{abstract}
Neuromyelitis optica (NMO), considered to be mediated by autoantibodies, often cause severely disabling disorders of the central nervous system, and predominantly cause optic nerve damage and longitudinally extensive transverse myelitis. Remarkable progress has been made in deciphering NMO pathogenesis during the past decade. In 2015, the International Panel for NMO Diagnosis proposed the unifying term "NMO spectrum disorders" (NMOSD) and the updated NMOSD criteria reflects a wide range of disease and maintains reasonable specificity. Moreover, cumulative findings have indicated that NMOSD are frequently associated with multiple autoimmune diseases, thereby presenting complex clinical symptoms that make this disease more difficult to recognize. Notably, most neurologists do not heed these symptoms or comorbid conditions in patients with NMOSD. Whereas previous reviews have focused on pathogenesis, treatment, and prognosis in NMOSD, we summarize the present knowledge with particular emphasis on atypical manifestations and autoimmune comorbidities in patients with NMOSD. Furthermore, we emphasized the identification of these atypical characteristics to enable a broader and better understanding of NMOSD, and improve early accurate diagnosis and therapeutic decision making.
\end{abstract}

Keywords: neuromyelitis optica spectrum disorders, anti-aquaporin-4 antibody, comorbid conditions, atypical manifestations

\section{Introduction}

Neuromyelitis optica (NMO) is an inflammatory disorder of the central nervous system (CNS), which typically presents with clinical signs of optic nerve damage and longitudinally extensive transverse myelitis. ${ }^{1-3}$ The discovery of aquaporin-4 immunoglobulin $\mathrm{G}$ antibody (AQP4-IgG) as a highly specific biomarker for NMO has substantially changed our understanding of NMO immunopathogenesis and revolutionized its diagnostic criteria. ${ }^{4-10}$ In 2015, the International Panel for NMO Diagnosis discarded the term "NMO" for a unifying term "NMO spectrum disorders" (NMOSD). ${ }^{5}$ Serum, and not cerebrospinal fluid AQP4 antibody, plays a central role in the diagnosis of NMOSD in clinical practice at present. ${ }^{11-13}$ The revised criteria encompass the core clinical characteristics, namely optic neuritis, acute myelitis, area postrema syndrome (episodes of otherwise unexplained hiccups, nausea, and vomiting), acute brainstem syndrome, symptomatic narcolepsy, or acute diencephalic clinical syndrome with NMOSD-typical diencephalic magnetic resonance imaging (MRI) lesions and a symptomatic cerebral syndrome with NMOSD-typical brain lesions. ${ }^{1,5}$ Moreover, the revised criteria enable the diagnosis of seronegative NMOSD when other supportive clinical and imaging features are present. ${ }^{5,14}$ More importantly, the updated NMOSD 
criteria reflects a wide range of disease and maintains reasonable specificity. Whereas unexplained hiccups, nausea, vomiting, and symptomatic narcolepsy were earlier considered atypical presentations of NMOSD, ${ }^{15,16}$ these constitute the core clinical characteristics in the present diagnostic criteria. ${ }^{5}$ There is widespread consensus that NMOSD are frequently associated with multiple autoimmune diseases, thereby presenting complex clinical symptoms. In this vein, we have begun to recognize several rare but possible manifestations and overlapping autoimmune diseases in patients with NMOSD. ${ }^{17}$ However, these symptoms and comorbid conditions in patients with NMOSD have not aroused much concern among clinical neurologists. This review attempts to present a new message and summarize the present state of knowledge on these significant atypical presentations and comorbid conditions in patients with NMOSD.

\section{What are the typical manifestations of NMOSD?}

Rationally, an understanding of the typical clinical features of NMOSD should precede a discussion of atypical presentations. NMOSD preferentially affect the optic nerve and spinal cord. Thus, acute myelitis and optic neuritis are the typical manifestations throughout the duration of the disease. It is highly possible that severe residual visual loss is particularly suggestive of NMOSD. Based on present diagnostic criteria, NMOSD could be further classified into NMOSD with or without AQP4 antibody. ${ }^{5}$ The updated and expanded criteria markedly improve the diagnostic yield by well reflecting a broader clinical spectrum of NMOSD, when compared with the previous criteria. ${ }^{12,18}$ Under the revised criteria, certain clinical manifestations are particularly suggestive of NMOSD, including area postrema clinical syndrome, simultaneous bilateral optic neuritis, optic neuritis that involves the chiasm, and complete transverse myelitis. ${ }^{19}$ In addition, clinical and laboratory "red flags" have been well-established to describe several features that signal the possibility of alternative diagnoses. ${ }^{5,20-23}$

\section{The complexity and wide range of NMOSD}

Increasing incidences of uncommon manifestations and signs in patients with NMOSD have been recently reported (Table 1). ${ }^{24-36}$ Although these are not considered to typify NMOSD in the present diagnostic criteria, these manifestations and signs may potentially promote a better understanding of this complex disease. A high frequency of brainstem symptoms could be observed in patients with NMOSD,
Table I Rare manifestations in patients with NMOSD

\begin{tabular}{|c|c|}
\hline Rare manifestations & References \\
\hline Brain cortical lesions & Tahara et al, ${ }^{45} \mathrm{Kim}$ et $\mathrm{al},{ }^{46} \mathrm{Han}$ et $\mathrm{a}^{48}$ \\
\hline Epileptic seizures & Cheng et al,,$^{59}$ Nakano et al ${ }^{60}$ \\
\hline \multirow[t]{3}{*}{ Intractable pruritus } & El Otmani et al, ${ }^{75}$ Xiao et al, ${ }^{76}$ \\
\hline & He et al, ${ }^{77}$ Netravathi et al, ${ }^{78}$ \\
\hline & Ramasamy et al $\mathrm{a}^{79}$ \\
\hline \multirow[t]{2}{*}{ Raynaud's phenomenon } & Sergio et al, ${ }^{82}$ Martin et al, ${ }^{83}$ \\
\hline & Franciotta et al, ${ }^{84}$ Jacobi et al ${ }^{85}$ \\
\hline Cervicogenic headache & Masters-Israilov and Robbins ${ }^{27}$ \\
\hline Bilateral hand edema & Sergio et $\mathrm{a}^{82}$ \\
\hline Erythematous rash & Cooper et $\mathrm{a}^{81}$ \\
\hline Pathologic laughing & Ahn et $\mathrm{a}^{25}$ \\
\hline Hemiageusia & Wang et $\mathrm{al}^{26}$ \\
\hline Trigeminal autonomic cephalalgia & Mathew et $\mathrm{al}^{71}$ \\
\hline Hydrocephalus & Clardy et $\mathrm{al}^{24}$ \\
\hline Sclerodactyly & Parperis ${ }^{80}$ \\
\hline Horner syndrome & Uludag et $\mathrm{al}^{28}$ \\
\hline Wernekink commissure & Zou and $\mathrm{Chen}^{29}$ \\
\hline \multicolumn{2}{|l|}{ syndrome } \\
\hline $\begin{array}{l}\text { Wall-eyed bilateral internuclear } \\
\text { ophthalmoplegia }\end{array}$ & Zou and Chen ${ }^{29}$ \\
\hline $\begin{array}{l}\text { ophthalmoplegia } \\
\text { Hearing loss }\end{array}$ & Tanaka and Tanaka ${ }^{30}$ \\
\hline \multirow[t]{2}{*}{ HyperCKemia } & He et al, ${ }^{31}$ lyer et al, ${ }^{36}$ Guo et al, ${ }^{35}$ \\
\hline & Abkur et $\mathrm{al}^{34}$ \\
\hline Paroxysmal sneezing & Lopez-Chiriboga et a $\left.\right|^{32}$ \\
\hline
\end{tabular}

Abbreviation: NMOSD, neuromyelitis optica spectrum disorders.

particularly vomiting, hiccups, oculomotor dysfunction, and pruritus, followed by hearing loss, facial palsy, vertigo, and vestibular and trigeminal neuralgia. ${ }^{37}$ Some atypical manifestations and comorbid conditions in patients with NMOSD will be discussed below, aiming to update the knowledge of clinical neurologists.

\section{Brain cortical lesions in NMOSD}

The pattern of brain lesions observed in NMOSD differs from that in multiple sclerosis (MS), and its appearance in characteristic locations facilitates the diagnosis of NMOSD. ${ }^{38,39}$ Pathologic and imaging studies sensitive to brain cortical lesions have revealed that these lesions are absent in Western patients with NMOSD. ${ }^{40-43}$ Previous studies have also reported that cognitive and cortical neuroimaging abnormalities in NMOSD are not attributable to cortical demyelination. ${ }^{44}$ To date, the absence of cortical lesions has been considered a "red flag" in the diagnosis of NMOSD. However, brain lesions that involve the cerebral cortex in NMOSD have been found in clinical practice among Asians, albeit very rarely. Tahara et al reported that three Japanese patients with anti-AQP4 antibody-positive NMOSD and encephalopathy-like symptoms exhibited brain cortical abnormalities on MRI, which were potentially attributable to the functional involvement of the cerebral cortex. ${ }^{45}$ Furthermore, 
Kim et al retrospectively analyzed 215 Korean NMOSD patients with AQP4 antibody. The infiltration of inflammatory cells across the damaged blood-brain barrier and leptomeningeal blood barrier into the adjacent cortex could be the possible explanation of brain cortical lesions in NMOSD. ${ }^{46,47}$ More recently, we noted identical findings and cortical demyelinating lesions could also be seen in a Chinese patient of NMOSD without AQP4 antibody. ${ }^{48}$ It was hypothesized that cortical demyelinating lesions in patients with NMOSD may be more commonly observed in East Asian populations than in Western populations, because the prevalence of NMOSD is considerably lower in regions that comprise mainly of Caucasians. Another possible explanation could be that differences exist with regard to the pathogenesis of NMOSD between patients of Eastern and Western origins.

More importantly, the possibility of alternative diagnoses must be considered by clinical neurologists. For example, NMOSD overlapping autoimmune encephalitis has been increasingly recognized and reported. ${ }^{49-51}$ Autoimmune encephalitis is also a severe inflammatory disorder mediated by antibodies against neuronal cell surface or synaptic proteins. Memory deficits, psychiatric symptoms, brain cortical lesions, behavioral disturbances, and decreased level of consciousness are typical of autoimmune encephalitis. ${ }^{52}$ In addition, alternative diagnoses such as Susac syndrome should also be ruled out during follow-up..$^{53}$ Furthermore, Luo et al reported on a patient with anti- $N$-methyl-D-aspartate receptor (NMDAR) encephalitis that occurred sequentially with NMOSD. The patient initially presented with cortical lesions in the brain MRI that bilaterally involved the medial temporal lobes. ${ }^{49}$ For this reason, the clinical diagnosis of NMOSD with brain cortical lesions should be considered with caution without excluding resembling diseases. Recognizing and accurately diagnosing autoimmune encephalitis coexisting with NMOSD, particularly patients who mainly present with brain cortical involvement, remain challenging for clinical neurologists. ${ }^{54}$ Further evaluations are needed to push the boundaries of NMOSD diagnosis through advanced MRI techniques or pathological investigations that reveal the characteristics of cortex involving lesions in NMOSD. ${ }^{55}$

\section{Epileptic seizures}

Epileptic seizures are not rare and have a greater incidence in patients with MS than in the general population. ${ }^{56-58}$ However, epileptic seizures as early symptoms are rarely presented in patients with NMOSD. For example, none of the patients developed any seizures in a study of 69 Chinese patients with
NMOSD, giving rise to speculations that seizures may be an atypical presentation in NMOSD. ${ }^{59}$ However, a contradictory finding has been reported that patients with NMOSD may possibly have a higher risk of developing epileptic seizures compared to the general population. ${ }^{60}$ Furthermore, Nakano et al reported higher expanded disability status scale scores for NMOSD patients with seizures, suggesting a poorer prognosis compared with patients without seizures. ${ }^{60}$ A possible explanation for the association between epileptic seizures and NMOSD is that severe prominent inflammation and axonal damage in NMOSD may cause epileptic seizures.

Furthermore, a subset of patients with NMOSD and positive for myelin oligodendrocyte glycoprotein (MOG) may further extend the spectrum of clinical phenotypes than we had previously thought. ${ }^{61-65}$ More recently, researchers have reported that MOG-IgG is closely correlated to generalized epileptic seizure and unilateral cerebral cortical encephalitis. ${ }^{66}$ Further studies are warranted to characterize the clinical features of epileptic seizures in patients with NMOSD, in order to reveal the mechanism underlying the development of epileptic seizures.

\section{Intractable pruritus}

Pruritus can be viewed as an unpleasant sensation which provokes a desire to scratch and relieve the symptom. ${ }^{67}$ Pruritus and pain have been known to closely interact. ${ }^{68} \mathrm{We}$ are beginning to reach the realization that sensory symptoms including neuropathic pain, painful tonic spasms, and trigeminal autonomic cephalalgia constitute presenting features in patients with NMOSD. ${ }^{69-74}$ However, the symptom of intractable pruritus in patients with NMOSD does not cause much concern among clinical neurologists. In fact, 12 patients reported pruritus during their illness in a welldefined cohort of 45 British patients with NMOSD. ${ }^{75}$ In this regard, Xiao et al retrospectively analyzed 64 Chinese NMOSD patients who were AQP4-IgG positive, and found that $28 \%(18 / 64)$ of these patients had the symptom of pruritus. ${ }^{76}$ Furthermore, recent studies have reported identical findings and further revealed that pruritus can be detected as an initial symptom before the attack. Hence, this may indicate a new episode of myelitis in patients with NMOSD. ${ }^{77,78}$ Although it has only been supported by case reports so far, intractable pruritus could be an underestimated characteristic feature of NMOSD. Several possible mechanisms could explain intractable pruritus as a symptom in patients with NMOSD. First, the inflammatory involvement of dorsal horn neurons in the spinal cord, spinal nucleus of the trigeminal nerve, or periaqueductal pathways may subsequently induce 
an itching sensation. ${ }^{77,79}$ Second, a partial demyelinating lesion insufficient to produce a permanent neurological deficit might cause minor irritation of the surrounding axons, leading to paroxysmal pruritus. This reflects the typical centralized location of lesions in the spinal cord that affects dorsal horn neurons and gray matter in patients with NMOSD. ${ }^{76} \mathrm{~A}$ third possible mechanism may be that neurons involved in the regulation of neuropathic pruritus may highly express AQP4, and thereby be preferentially involved in NMOSD. ${ }^{75}$

\section{Cutaneous manifestations}

Nonspecific cutaneous manifestations such as erythematous rash, sclerodactyly, and bilateral edema of the hands have been noted in the setting of NMOSD, although these have been limited to isolated case reports. ${ }^{80-82}$ Among these, Raynaud's phenomenon was the most common cutaneous presentation in patients with NMOSD. ${ }^{82-85}$ These case studies highlight the importance of the early recognition of cutaneous signs, which may help optimize clinical neurologists' decision making. Martin et al reported the case of a 60-year-old Chinese patient who initially presented with nonspecific cutaneous findings such as Gottron's papules and bilateral hand edema. However, it was finally found that the patient had features of overlapping autoimmune diseases, including rheumatoid arthritis and amyopathic dermatomyositis in the setting of NMOSD with AQP4-IgG.$^{83}$ Furthermore, Delman et al recently reported dermatomyositis as a clinical presentation in a 40-year-old Caucasian NMOSD patient with AQP4-IgG. The patient also tested positive for antimelanoma differentiation-associated gene 5 antibody, which is one of the most common dermatomyositis-specific antibodies. ${ }^{86}$ Therefore, clinical neurologists must focus more attention on these clinical features and antibody testing for additional overlapping autoimmune diseases.

\section{NMOSD coexisting with subacute combined degeneration}

NMOSD is often idiopathic, although it has been strongly associated with other autoimmune diseases and non-organspecific autoantibodies(Table 2). ${ }^{34-36,87-101}$ Apparently, NMOSD is not related to subacute combined degeneration to some extent. Interestingly, Ishii et al reported the case of a 36-yearold Japanese woman who simultaneously had NMOSD with AQP4-IgG and subacute combined degeneration. ${ }^{102}$ Cervical MRI revealed that long cord lesions around the central spinal canal were typical for NMOSD. MRI scans also revealed bilateral symmetric hyperintense signals in posterior columns, which are the so-called "inverted V" sign in patients
Table 2 Coexisting conditions associated with NMOSD

\begin{tabular}{|c|c|}
\hline Comorbid conditions & References \\
\hline Subacute combined degeneration & Ishii et al ${ }^{102}$ \\
\hline Autoimmune encephalitis & Luo et al, ${ }^{49}$ Qin et al, ${ }^{50}$ Ran et al ${ }^{51}$ \\
\hline Idiopathic intracranial hypertension & Viswanathan and Wong ${ }^{87}$ \\
\hline Hematological immune disease & Patejdl et al ${ }^{88}$ \\
\hline Thrombopenic purpura & Wang et $\mathrm{al}^{89}$ \\
\hline Hypertrophic pachymeningitis & Kon et a $\left.\right|^{90}$ \\
\hline $\begin{array}{l}\text { Postural orthostatic tachycardia } \\
\text { syndrome }\end{array}$ & Barun et $\mathrm{al}^{91}$ \\
\hline Osmotic demyelination syndrome & Adamec et al ${ }^{92}$ \\
\hline $\begin{array}{l}\text { Posterior reversible } \\
\text { encephalopathy syndrome }\end{array}$ & Magana et al $^{93}$ \\
\hline $\begin{array}{l}\text { Autoimmune lymphoproliferative } \\
\text { syndrome }\end{array}$ & Cooper et $\mathrm{al}^{81}$ \\
\hline Systemic sclerosis & Pereira et $\mathrm{al}^{94}$ \\
\hline Systemic lupus erythematosus & Pereira et al,${ }^{94}$ Asgari et al ${ }^{95}$ \\
\hline Sjögren's syndrome & $\begin{array}{l}\text { Zhong et al, }{ }^{96} \text { Kahlenberg, }{ }^{108} \\
\text { Jayarangaiah et al, }{ }^{109} \text { Qiao et al,"' } \\
\text { Birnbaum et al }{ }^{112}\end{array}$ \\
\hline Mixed connective tissue & Parperis $^{80}$ \\
\hline Hashimoto thyroiditis & Pereira et a $\left.\right|^{94}$ \\
\hline Myasthenia gravis & Ikeguchi et al, ${ }^{97}$ Jarius et al ${ }^{101}$ \\
\hline Dermatomyositis & Martin et $\mathrm{al}^{83}$ \\
\hline Bacterial meningomyelitis & Li et al ${ }^{98}$ \\
\hline Celiac disease & Matijaca et $\mathrm{a}^{99}$ \\
\hline Spinocerebellar ataxia type 3I & Takahashi et al ${ }^{100}$ \\
\hline
\end{tabular}

Abbreviation: NMOSD, neuromyelitis optica spectrum disorders.

with subacute combined degeneration. ${ }^{102}$ Furthermore, vitamin B12 deficiency may be associated with AQP4-IgG in this patient, in view of no prior history of gastrointestinal disorders. Recent studies have highlighted that low levels of vitamin B12 were found in NMOSD patients with AQP4IgG. ${ }^{103}$ It is a well-established fact that AQP4 is not only mainly expressed in astrocytes within the CNS, but also in diverse organs such as the stomach, skeletal muscle, inner ear, and kidneys. ${ }^{17,104}$ Thus, AQP4-IgG may inhibit the production of intrinsic factor and gastric acid secretion by parietal cells with resultant vitamin B12 malabsorption. In summary, the presence of AQP4 receptors outside the CNS may help explain some of the unusual features of NMOSD. It is highly desirable that physicians note this atypical phenomenon in clinical practice.

\section{NMOSD coexisting with anti-NMDAR encephalitis}

Anti-NMDAR encephalitis is also a severe autoimmune disease that has been commonly associated with the production of antibodies against NMDAR..$^{52,105}$ NMOSD in coexistence with anti-NMDAR encephalitis is relatively rare, but cannot be ignored as a possibility. ${ }^{106} \mathrm{~A}$ previous study revealed that NMDAR autoimmunity is in fact not involved in NMOSD 
because a total of 98 NMOSD patients with AQP4-IgG showed negative results for NMDAR antibodies. ${ }^{107}$ However, recent studies have inferred the opposite conclusion that NMOSD overlapping anti-NMDAR encephalitis is increasingly noted, as evidenced by the simultaneous detection of both NMDAR antibodies and AQP4-IgG in serum samples. ${ }^{49-51}$ In 2017 , Ran et al succinctly summarized the clinical characteristics of 34 patients who presented with both NMOSD and anti-NMDAR encephalitis. ${ }^{51}$ Among these patients, some who presented with obvious symptoms of anti-NMDAR encephalitis at onset subsequently manifested concurrent or separate episodes of NMOSD during the following time. Conversely, some patients with NMOSD in the early stages subsequently presented with anti-NMDAR encephalitis, while others simultaneously suffered from NMOSD and anti-NMDAR encephalitis during the disease period. The possibility of a direct or indirect connection between these two immune-mediated disorders was also proposed, which predisposes its susceptibility to other autoimmune-associated disorders. ${ }^{51}$ Further studies are still needed to thoroughly investigate the association between NMOSD and anti-NMDAR encephalitis.

\section{NMOSD coexisting with Sjögren's syndrome (SS)}

SS is a chronic systemic illness typically characterized by the dysfunction and inflammatory infiltration of the exocrine glands. Although it has been increasingly recognized that SS mimics and coexists with NMOSD, distinguishing between these two disorders has been proven to be difficult for neurologists and rheumatologists. ${ }^{108,109}$ Specifically, patients with SS can commonly experience extraglandular manifestations such as CNS involvement, and appear as transverse myelitis, optic neuritis, and brain abnormalities, which complicate the diagnosis. ${ }^{110}$ Qiao et al retrospectively analyzed 616 patients with SS in a Chinese single center study and found that $7 \%(43 / 616)$ of these patients had coexisting NMOSD. Based on this finding, they hypothesized that NMOSD and SS in fact share common pathophysiological features and that NMOSD is a neurological complication of SS. ${ }^{111}$ However, contradictory findings have been reported that the complex relationship between NMOSD and SS can be attributed to co-occurring autoimmunity, instead of directly implicating CNS involvement in patients with SS. Researchers revealed that AQP4 antibodies were only detected in SS with NMOSD versus non-NMOSD patients with SS. ${ }^{112}$ In addition, AQP5 rather than AQP4 contributes to salivary secretion in SS patients with NMOSD. ${ }^{113}$
Moreover, another recent study found that patients with NMOSD and SS have higher non-organ-specific antibodies and enhanced autoimmune responses compared to non-SS patients with NMOSD ${ }^{96}$ Hence, only sufficient knowledge of NMOSD patients with and without SS could facilitate prompt recognition and decision making for this condition.

\section{Conclusion}

Some atypical manifestations and comorbid conditions in NMOSD have been reported as rare events, but may represent a broader spectrum of symptoms, compared to those previously described in conventional descriptions. The awareness that NMOSD is frequently associated with other autoimmune diseases is important to facilitate specific antibody testing when diagnosing cases with an associated autoimmune disease. Ruling out other entities during follow-up is of importance for clinical neurologists. Future studies with larger NMOSD cohorts may confirm the accumulated knowledge base from these case series, and identify additional clues with regard to the heterogeneous features of NMOSD. This calls for greater efforts in identifying its etiopathogenic mechanisms in NMOSD patients with atypical manifestations and comorbidities, in order to broaden our knowledge of this complex disease spectrum. We believe that research on NMOSD in the next decade will promise to be as exciting as that in the past decade.

\section{Acknowledgments}

This work was supported by grants from the General Program of the National Natural Science Foundation of China (nos 81671177 and 81471216), the International Science and Technology Cooperation Program of Jilin Provincial Science and Technology Development of China (no 20150414011GH), the Technology Innovation Program of Jilin Provincial Health and Family Planning Commission of China (no 2016J040), the Norman Bethune Cultivation Plan of Jilin University (no 2015320), the Science and Technology Program of Jilin Provincial Education Department of China (no 2016-462), the Swedish Research Council (no 201503005), and the First Hospital, Jilin University, China. We gratefully thank Majid Pahlevan Kakhki of Tarbiat Modares University, for his help in revising this manuscript.

\section{Disclosure}

The authors report no conflicts of interest in this work.

\section{References}

1. Weinshenker BG, Wingerchuk DM. Neuromyelitis spectrum disorders. Mayo Clin Proc. 2017;92(4):663-679. 
2. Han J, Sun L, Wang Z, et al. Circulating regulatory B cell subsets in patients with neuromyelitis optica spectrum disorders. Neurol Sci. 2017;38(7):1205-1212.

3. Jasiak-Zatonska M, Kalinowska-Lyszczarz A, Michalak S, Kozubski W. The immunology of neuromyelitis optica - current knowledge, clinical implications, controversies and future perspectives. Int J Mol Sci. 2016;17(3):273.

4. Lennon VA, Wingerchuk DM, Kryzer TJ, et al. A serum autoantibody marker of neuromyelitis optica: distinction from multiple sclerosis. Lancet. 2004;364(9451):2106-2112.

5. Wingerchuk DM, Banwell B, Bennett JL, et al. International consensus diagnostic criteria for neuromyelitis optica spectrum disorders. Neurology. 2015;85(2):177-189.

6. Wingerchuk DM, Lennon VA, Pittock SJ, Lucchinetti CF, Weinshenker BG. Revised diagnostic criteria for neuromyelitis optica. Neurology. 2006;66(10):1485-1489.

7. Kim Y, Kim G, Kong BS, et al. Large-scale in-house cell-based assay for evaluating the serostatus in patients with neuromyelitis optica spectrum disorder based on new diagnostic criteria. J Clin Neurol. 2017; 13(2):175-180.

8. Yang X, Ransom BR, Ma JF. The role of AQP4 in neuromyelitis optica: more answers, more questions. J Neuroimmunol. 2016;298:63-70.

9. Zekeridou A, Lennon VA. Aquaporin-4 autoimmunity. Neurol Neuroimmunol Neuroinflamm. 2015;2(4):e110.

10. Metz I, Beissbarth T, Ellenberger D, et al. Serum peptide reactivities may distinguish neuromyelitis optica subgroups and multiple sclerosis. Neurol Neuroimmunol Neuroinflamm. 2016;3(2):e204.

11. Whittam D, Wilson M, Hamid S, Keir G, Bhojak M, Jacob A. What's new in neuromyelitis optica? A short review for the clinical neurologist. J Neurol. Epub 2017 Mar 13.

12. Hyun JW, Jeong IH, Joung A, Kim SH, Kim HJ. Evaluation of the 2015 diagnostic criteria for neuromyelitis optica spectrum disorder. Neurology. 2016;86(19):1772-1779.

13. Majed M, Fryer JP, McKeon A, Lennon VA, Pittock SJ. Clinical utility of testing AQP4-IgG in CSF: guidance for physicians. Neurol Neuroimmunol Neuroinflamm. 2016;3(3):e231.

14. Uzawa A, Mori M, Uchida T, Masuda H, Ohtani R, Kuwabara S. Seronegative neuromyelitis optica spectrum disorder patients diagnosed using new diagnostic criteria. Mult Scler. 2016;22(10):1371-1375.

15. Sato D, Fujihara K. Atypical presentations of neuromyelitis optica. Arq Neuropsiquiatr. 2011;69(5):824-828.

16. Yavuz H, Kiresi D. Unusual manifestations of pediatric neuromyelitis optica. J Child Neurol. 2013;28(5):658-662.

17. Rosales D, Kister I. Common and rare manifestations of neuromyelitis optica spectrum disorder. Curr Allergy Asthma Rep. 2016;16(6):42.

18. Hamid SH, Elsone L, Mutch K, Solomon T, Jacob A. The impact of 2015 neuromyelitis optica spectrum disorders criteria on diagnostic rates. Mult Scler. 2017;23(2):228-233.

19. Ramanathan S, Prelog K, Barnes EH, et al. Radiological differentiation of optic neuritis with myelin oligodendrocyte glycoprotein antibodies, aquaporin-4 antibodies, and multiple sclerosis. Mult Scler. 2016;22(4):470-482.

20. Jarius S, Paul F, Franciotta D, et al. Cerebrospinal fluid findings in aquaporin-4 antibody positive neuromyelitis optica: results from 211 lumbar punctures. J Neurol Sci. 2011;306(1-2):82-90.

21. Kitley JL, Leite MI, George JS, Palace JA. The differential diagnosis of longitudinally extensive transverse myelitis. Mult Scler. 2012;18(3): 271-285.

22. Trebst C, Raab P, Voss EV, et al. Longitudinal extensive transverse myelitis - it's not all neuromyelitis optica. Nat Rev Neurol. 2011;7(12): 688-698.

23. Wingerchuk DM, Pittock SJ, Lucchinetti CF, Lennon VA, Weinshenker BG. A secondary progressive clinical course is uncommon in neuromyelitis optica. Neurology. 2007;68(8):603-605.

24. Clardy SL, Lucchinetti CF, Krecke KN, et al. Hydrocephalus in neuromyelitis optica. Neurology. 2014;82(20):1841-1843.
25. Ahn SW, Kim JM, Park KY, Kwon OS, Youn YC. Pathologic laughing as a presenting feature in a patient with neuromyelitis optica. J Neuropsychiatry Clin Neurosci. 2014;26(2):E58-E59.

26. Wang R, Qi D, Zhang Y. Neuromyelitis optica spectrum disorder initiated with hemiageusia and pruritus: a case report. J Neurol Sci. 2015; 348(1-2):288-289.

27. Masters-Israilov A, Robbins MS. Headache in neuromyelitis optica. Curr Pain Headache Rep. 2017;21(4):20.

28. Uludag IF, Sariteke A, Ocek L, et al. Neuromyelitis optica presenting with horner syndrome: a case report and review of literature. Mult Scler Relat Disord. 2017;14:32-34.

29. Zou Z, Chen W. Can Wernekink commissure syndrome and wall-eyed bilateral internuclear ophthalmoplegia be ascribed to neuromyelitis optica spectrum disorder? Neurol Sci. Epub 2017 Jul 5.

30. Tanaka M, Tanaka K. Sudden hearing loss as the initial symptom in Japanese patients with multiple sclerosis and seropositive neuromyelitis optica spectrum disorders. J Neuroimmunol. 2016;298:16-18.

31. He D, Li Y, Dai Q, et al. Myopathy associated with neuromyelitis optica spectrum disorders. Int J Neurosci. 2016;126(10):863-866.

32. Lopez-Chiriboga AS, Huang JF, Flanagan EP, Cheshire WP Jr. Paroxysmal sneezing in NMOSD: further evidence of the localization of the human sneeze center. Neurol Neuroimmunol Neuroinflamm. 2017; 4(1):e303.

33. Jarius S, Lauda F, Wildemann B, Tumani H. Steroid-responsive hearing impairment in NMO-IgG/aquaporin-4-antibody-positive neuromyelitis optica. J Neurol. 2013;260(2):663-664.

34. Abkur TM, Foran E, Kearney H, Harkin G, Byrnes V, Lynch J. Neuromyelitis optica presenting as intractable vomiting and hyperCKaemia. J Neurol. 2016;263(1):171-173.

35. Guo Y, Lennon VA, Popescu BF, et al. Autoimmune aquaporin-4 myopathy in neuromyelitis optica spectrum. JAMA Neurol. 2014;71(8): 1025-1029.

36. Iyer A, Rathnasabapathi D, Elsone L, et al. Transverse myelitis associated with an itchy rash and hyperckemia: neuromyelitis optica associated with dermatitis herpetiformis. JAMA Neurol. 2014;71(5):630-633.

37. Kremer L, Mealy M, Jacob A, et al. Brainstem manifestations in neuromyelitis optica: a multicenter study of 258 patients. Mult Scler. 2014;20(7):843-847.

38. Pittock SJ, Lennon VA, Krecke K, Wingerchuk DM, Lucchinetti CF, Weinshenker BG. Brain abnormalities in neuromyelitis optica. Arch Neurol. 2006;63(3):390-396.

39. Kim HJ, Paul F, Lana-Peixoto MA, et al. MRI characteristics of neuromyelitis optica spectrum disorder: an international update. Neurology. 2015;84(11):1165-1173.

40. Calabrese M, Oh MS, Favaretto A, et al. No MRI evidence of cortical lesions in neuromyelitis optica. Neurology. 2012;79(16):1671-1676.

41. Popescu BF, Parisi JE, Cabrera-Gomez JA, et al. Absence of cortical demyelination in neuromyelitis optica. Neurology. 2010;75(23): 2103-2109.

42. Sinnecker T, Dorr J, Pfueller CF, et al. Distinct lesion morphology at 7-T MRI differentiates neuromyelitis optica from multiple sclerosis. Neurology. 2012;79(7):708-714.

43. Kister I, Herbert J, Zhou Y, Ge Y. Ultrahigh-field MR (7 T) imaging of brain lesions in neuromyelitis optica. Mult Scler Int. 2013;2013: 398259.

44. Liu Y, Xie T, He Y, et al. Cortical thinning correlates with cognitive change in multiple sclerosis but not in neuromyelitis optica. Eur Radiol. 2014;24(9):2334-2343.

45. Tahara M, Ito R, Tanaka K, Tanaka M. Cortical and leptomeningeal involvement in three cases of neuromyelitis optica. Eur JNeurol. 2012; 19(5):e47-e48.

46. Kim W, Lee JE, Kim SH, et al. Cerebral cortex involvement in neuromyelitis optica spectrum disorder. J Clin Neurol. 2016;12(2):188-193.

47. Asgari N, Flanagan EP, Fujihara K, et al. Disruption of the leptomeningeal blood barrier in neuromyelitis optica spectrum disorder. Neurol Neuroimmunol Neuroinflamm. 2017;4(4):e343. 
48. Han J, Guo C, Lin C, et al. Cortical demyelinating lesions in neuromyelitis optica spectrum disorders? A case study with 8-year follow-up. Int J Clin Exp Med. 2017;10(1):1461-1464.

49. Luo JJ, Lv H, Sun W, et al. Anti-N-methyl-D-aspartate receptor encephalitis in a patient with neuromyelitis optica spectrum disorders. Mult Scler Relat Disord. 2016;8:74-77.

50. Qin K, Wu W, Huang Y, et al. Anti-N-methyl-D-aspartate receptor (NMDAR) antibody encephalitis presents in atypical types and coexists with neuromyelitis optica spectrum disorder or neurosyphilis. BMC Neurol. 2017;17(1):1.

51. Ran Y, Wang L, Zhang F, Ao R, Dong Z, Yu S. Anti-NMDAR encephalitis followed by seropositive neuromyelitis optica spectrum disorder: a case report and literature review. Clin Neurol Neurosurg. 2017;155: 75-82.

52. Leypoldt F, Armangue T, Dalmau J. Autoimmune encephalopathies. Ann N Y Acad Sci. 2015;1338:94-114.

53. Dorr J, Krautwald S, Wildemann B, et al. Characteristics of Susac syndrome: a review of all reported cases. Nat Rev Neurol. 2013;9(6) 307-316.

54. Ramberger $M$, Bsteh $\mathrm{G}$, Schanda $\mathrm{K}$, et al. NMDA receptor antibodies: a rare association in inflammatory demyelinating diseases. Neurol Neuroimmunol Neuroinflamm. 2015;2(5):e141.

55. Kremer S, Renard F, Achard S, et al. Use of advanced magnetic resonance imaging techniques in neuromyelitis optica spectrum disorder JAMA Neurol. 2015;72(7):815-822.

56. Lund C, Nakken KO, Edland A, Celius EG. Multiple sclerosis and seizures: incidence and prevalence over 40 years. Acta Neurol Scand. 2014;130(6):368-373.

57. Etemadifar M, Abtahi SH, Roomizadeh P. Epileptic seizures in multiple sclerosis: a population-based survey in Iran. Acta Neurol Belg. 2013;113(3):271-278.

58. Lapato AS, Szu JI, Hasselmann JP, Khalaj AJ, Binder DK, TiwariWoodruff SK. Chronic demyelination-induced seizures. Neuroscience. 2017;346:409-422.

59. Cheng MY, Wai YY, Ro LS, Wu T. Seizures and multiple sclerosis in Chinese patients: a clinical and magnetic resonance imaging study. Epilepsy Res. 2012;101(1-2):166-173.

60. Nakano H, Tanaka M, Kinoshita M, et al. Epileptic seizures in Japanese patients with multiple sclerosis and neuromyelitis optica. Epilepsy Res. 2013;104(1-2):175-180.

61. Bernard-Valnet R, Liblau RS, Vukusic S, Marignier R. Neuromyelitis optica: a positive appraisal of seronegative cases. Eur J Neurol. 2015; 22(12):1511-1518

62. Kim SM, Woodhall MR, Kim JS, et al. Antibodies to MOG in adults with inflammatory demyelinating disease of the CNS. Neurol Neuroimmunol Neuroinflamm. 2015;2(6):e163.

63. Jarius S, Ruprecht K, Kleiter I, et al. MOG-IgG in NMO and related disorders: a multicenter study of 50 patients. Part 1: frequency, syndrome specificity, influence of disease activity, long-term course, association with AQP4-IgG, and origin. J Neuroinflammation. 2016;13(1):279.

64. Jarius S, Ruprecht K, Kleiter I, et al. MOG-IgG in NMO and related disorders: a multicenter study of 50 patients. Part 2: epidemiology, clinical presentation, radiological and laboratory features, treatment responses, and long-term outcome. J Neuroinflammation. 2016; 13(1):280

65. Zamvil SS, Slavin AJ. Does MOG Ig-positive AQP4-seronegative opticospinal inflammatory disease justify a diagnosis of NMO spectrum disorder? Neurol Neuroimmunol Neuroinflamm. 2015;2(1):e62.

66. Ogawa R, Nakashima I, Takahashi T, et al. MOG antibody-positive, benign, unilateral, cerebral cortical encephalitis with epilepsy. Neurol Neuroimmunol Neuroinflamm. 2017;4(2):e322.

67. Zhao S, Mutch K, Elsone L, Miller J, Jacob A. An unusual case of 'itchy paralysis': neuromyelitis optica presenting with severe neuropathic itch. Pract Neurol. 2015;15(2):149-151.

68. Nedelec B, Carrougher GJ. Pain and pruritus postburn injury. J Burn Care Res. 2017;38(3):142-145.
69. Carnero Contentti E, Leguizamon F, Hryb JP, et al. Neuromyelitis optica: association with paroxysmal painful tonic spasms. Neurologia. 2016;31(8):511-515.

70. Zhao S, Mutch K, Elsone L, Nurmikko T, Jacob A. Neuropathic pain in neuromyelitis optica affects activities of daily living and quality of life. Mult Scler. 2014;20(12):1658-1661.

71. Mathew T, Nadimpally US, Sarma GR, Nadig R. Trigeminal autonomic cephalalgia as a presenting feature of neuromyelitis optica: "a rare combination of two uncommon disorders". Mult Scler Relat Disord. 2016; 6:73-74.

72. Kong Y, Okoruwa H, Revis J, et al. Pain in patients with transverse myelitis and its relationship to aquaporin 4 antibody status. J Neurol Sci. 2016;368:84-88.

73. Bradl M, Kanamori Y, Nakashima I, et al. Pain in neuromyelitis opticaprevalence, pathogenesis and therapy. Nat Rev Neurol. 2014;10(9): 529-536.

74. Chavarro VS, Mealy MA, Simpson A, et al. Insufficient treatment of severe depression in neuromyelitis optica spectrum disorder. Neurol Neuroimmunol Neuroinflamm. 2016;3(6):e286.

75. El Otmani H, Dany F, El Moutawakil B, Abdoh-Rafai M, Slassi I. Intractable hiccup and vomiting, neuropathic pruritus and tonic spasms in a case of neuromyelitis optica spectrum disorder. Acta Neurol Belg. 2015;115(4):797-799.

76. Xiao L, Qiu W, Lu Z, Li R, Hu X. Intractable pruritus in neuromyelitis optica. Neurol Sci. 2016;37(6):949-954.

77. He Z, Ren M, Wang X, Guo Q, Qi X. Pruritus may be a common symptom related to neuromyelitis optica spectrum disorders. Mult Scler Relat Disord. 2017;13:1-3.

78. Netravathi M, Saini J, Mahadevan A, et al. Is pruritus an indicator of aquaporin-positive neuromyelitis optica? Mult Scler. 2017;23(6):810-817.

79. Ramasamy B, Kalidoss R, Gnanashanmugham G, Venkatesan EP Pruritus: is it a predictor of relapse in neuromyelitis optica spectrum disorder? Neurol India. 2014;62(3):333-334.

80. Parperis K. A rare case of mixed connective tissue disease complicated with neuromyelitis optica. J Clin Rheumatol. 2012;18(5):261-262.

81. Cooper N, Te Water Naude J, Connor PP. Neuromyelitis optica complicating autoimmune lymphoproliferative syndrome in a 4-year-old girl. Br J Haematol. 2011;152(3):247.

82. Sergio P, Mariana B, Alberto O, et al. Association of neuromyelitis optic (NMO) with autoimmune disorders: report of two cases and review of the literature. Clin Rheumatol. 2010;29(11):1335-1338.

83. Martin C, Maurer T, Mutizwa MM. Neuromyelitis optica with cutaneous findings: case report and review of the literature. Dermatology. 2015;230(4):289-292.

84. Franciotta D, Zardini E, Caporali R, et al. Systemic sclerosis in aquaporin-4 antibody-positive longitudinally extensive transverse myelitis. J Neurol Sci. 2011;303(1-2):139-141.

85. Jacobi C, Stingele K, Kretz R, et al. Neuromyelitis optica (Devic's syndrome) as first manifestation of systemic lupus erythematosus. Lupus 2006;15(2):107-109.

86. Delman D, Peng X, Zedek DC, Jewells V, Chahin N, Markovic-Plese S. Dermatomyositis as a presentation of neuromyelitis optica spectrum disorder. J Neuroimmunol. 2015;278:108-111.

87. Viswanathan S, Wong LC. A curious case of neuromyelitis optica spectrum disorder co-existing with idiopathic intracranial hypertension. J Clin Neurosci. 2017;41:104-106.

88. Patejdl R, Wittstock M, Zettl UK, Jost K, Grossmann A, Prudlo J. Neuromyelitis optica spectrum disorder coinciding with hematological immune disease: a case report. Mult Scler Relat Disord. 2016;9: 101-103.

89. Wang Y, Gong Q, Zhu M, et al. Aquaporin-4 positive neuromyelitis optica spectrum disorders secondary to thrombopenic purpura: a case report. Medicine. 2017;96(2):e5792.

90. Kon T, Nishijima H, Haga R, et al. Hypertrophic pachymeningitis accompanying neuromyelitis optica spectrum disorder: a case report. J Neuroimmunol. 2015;287:27-28. 
91. Barun B, Adamec I, Lovric M, Habek M. Postural orthostatic tachycardia syndrome: additional phenotypic feature of neuromyelitis optica spectrum disorder. Neurol Sci. 2014;35(10):1623-1625.

92. Adamec I, Kersic F, Crnosija L, Habek M. Neuromyelitis optica spectrum disorder associated with osmotic demyelination syndrome. Neurol Sci. 2016;37(6):1009-1011.

93. Magana SM, Matiello M, Pittock SJ, et al. Posterior reversible encephalopathy syndrome in neuromyelitis optica spectrum disorders. Neurology. 2009;72(8):712-717.

94. Pereira W, Reiche EMV, Kallaur AP, et al. Frequency of autoimmune disorders and autoantibodies in patients with neuromyelitis optica. Acta Neuropsychiatr. 2017;29(3):170-178.

95. Asgari N, Jarius S, Laustrup H, et al. Aquaporin-4-autoimmunity in patients with systemic lupus erythematosus: a predominantly population-based study. Mult Scler. Epub 2017 Mar 1.

96. Zhong YH, Zhong ZG, Zhou Z, et al. Comparisons of presentations and outcomes of neuromyelitis optica patients with and without Sjogren's syndrome. Neurol Sci. 2017;38(2):271-277.

97. Ikeguchi R, Shimizu Y, Suzuki S, et al. Japanese cases of neuromyelitis optica spectrum disorder associated with myasthenia gravis and a review of the literature. Clin Neurol Neurosurg. 2014;125:217-221.

98. Li X, Lin J, Pan S, et al. Suspected bacterial meningomyelitis: the first presenting clinical feature of neuromyelitis optica spectrum disorder. J Neuroimmunol. 2017;309:68-71.

99. Matijaca M, Pavelin S, Kaliterna DM, Bojic L, Matijaca A. Pathogenic role of aquaporin antibodies in the development of neuromyelitis optica in a woman with celiac disease. Isr Med Assoc J. 2011;13(3): 182-184.

100. Takahashi Y, Manabe Y, Morihara R, Narai H, Yamashita T, Abe K. Neuromyelitis optica spectrum disorder coinciding with spinocerebellar ataxia type 31. Case Rep Neurol. 2017;9(2):127-130.

101. Jarius S, Paul F, Franciotta D, et al. Neuromyelitis optica spectrum disorders in patients with myasthenia gravis: ten new aquaporin-4 antibody positive cases and a review of the literature. Mult Scler. 2012; 18(8):1135-1143.

102. Ishii N, Mochizuki H, Takahashi T, Shiomi K, Nakazato M. A case of simultaneous neuromyelitis optica spectrum disorder and subacute combined degeneration. Neurol Sci. 2013;34(10):1819-1821.
103. Jarius S, Paul F, Ruprecht K, Wildemann B. Low vitamin B12 levels and gastric parietal cell antibodies in patients with aquaporin-4 antibody-positive neuromyelitis optica spectrum disorders. J Neurol. 2012;259(12):2743-2745.

104. Papadopoulos MC, Verkman AS. Aquaporin 4 and neuromyelitis optica. Lancet Neurol. 2012;11(6):535-544.

105. Kayser MS, Dalmau J. Anti-NMDA receptor encephalitis, autoimmunity, and psychosis. Schizophr Res. 2016;176(1):36-40.

106. Liu CY, Zhu J, Zheng XY, Ma C, Wang X. Anti-N-methyl-D-aspartate receptor encephalitis: a severe, potentially reversible autoimmune encephalitis. Mediators Inflamm. 2017;2017:6361479.

107. Xie L, Long Y, Yang N, et al. No overlap among serum GAD65, NMDAR and AQP4 antibodies in patients with neuromyelitis optica spectrum disorders. Neuroimmunomodulation. 2015;22(5):337-341.

108. Kahlenberg JM. Neuromyelitis optica spectrum disorder as an initial presentation of primary Sjogren's syndrome. Semin Arthritis Rheum. 2011;40(4):343-348.

109. Jayarangaiah A, Sehgal R, Epperla N. Sjogren's syndrome and neuromyelitis optica spectrum disorders (NMOSD) - a case report and review of literature. BMC Neurol. 2014;14:200.

110. Kim SM, Kim SJ, Lee HJ, Kuroda H, Palace J, Fujihara K. Differential diagnosis of neuromyelitis optica spectrum disorders. Ther Adv Neurol Disord. 2017;10(7):265-289.

111. Qiao L, Wang Q, Fei Y, et al. The clinical characteristics of primary Sjogren's syndrome with neuromyelitis optica spectrum disorder in China: a STROBE-compliant article. Medicine. 2015;94(28):e1145.

112. Birnbaum J, Atri NM, Baer AN, Cimbro R, Montagne J, CasciolaRosen L. Relationship between neuromyelitis optica spectrum disorder and Sjogren's syndrome: central nervous system extraglandular disease or unrelated, co-occurring autoimmunity? Arthritis Care Res. 2017;69(7):1069-1075.

113. Yoshimura S, Nakamura H, Horai Y, et al. Abnormal distribution of AQP5 in labial salivary glands is associated with poor saliva secretion in patients with Sjogren's syndrome including neuromyelitis optica complicated patients. Mod Rheumatol. 2016;26(3):384-390.
Neuropsychiatric Disease and Treatment

\section{Publish your work in this journal}

Neuropsychiatric Disease and Treatment is an international, peerreviewed journal of clinical therapeutics and pharmacology focusing on concise rapid reporting of clinical or pre-clinical studies on a range of neuropsychiatric and neurological disorders. This journal is indexed on PubMed Central, the 'PsycINFO' database and CAS,

\section{Dovepress}

and is the official journal of The International Neuropsychiatric Association (INA). The manuscript management system is completely online and includes a very quick and fair peer-review system, which is all easy to use. Visit http://www.dovepress.com/testimonials.php to read real quotes from published authors. 